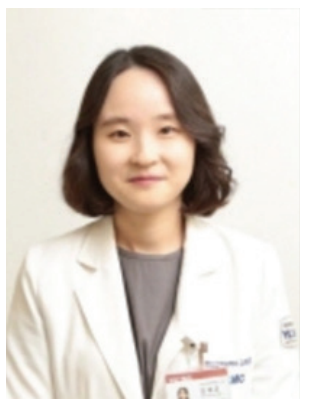

Received: August 19, 2021 Revised: August 26, 2021 Accepted: August 31, 2021

Corresponding author: Hye-Geum Kim, MD, PhD Department of Psychiatry, Yeungnam University College of Medicine, 170 Hyeonchung-ro, Nam-gu, Daegu 42415, Korea Tel: +82-53-620-3340 Fax: +82-53-657-3921

E-mail: psykhg@yu.ac.kr

\section{Understanding sleep and sleep disturbances in autism spectrum disorder, and management of insomnia: an update}

\author{
Hye-Geum Kim \\ Department of Psychiatry, Yeungnam University College of Medicine, Daegu, Korea
}

We spend 25 to 30 years sleeping in our lifetime. This time-consuming activity plays an important role in the quality of life. Chronic sleep disturbance is a pandemic worldwide, with two-thirds of individuals unable to obtain the recommended 7 to 9 hours of sleep each night. In addition, up to $10 \%$ of people meet the criteria for clinical insomnia [1]. The cost of insomnia is estimated to reach $\$ 100$ billion annually in the United States alone. Therefore, in this special issue, we attempt to understand the importance of sleep from several aspects: (1) understanding sleep, which affects not only mental health but also overall physical health [2]; (2) understanding the characteristics of sleep disturbances that occur in autism spectrum disorder (ASD) mainly in children [3]; and (3) new non-pharmacological treatments for insomnia [4].

Insomnia is treated as a neuropsychiatric disorder and its influence was thought to be confined to neurocognitive and emotional problems. However, insomnia affects the entire body and causes a variety of physical illnesses. Yun and Jo [2] reviewed the aspects of insomnia as a systemic disease. Chronic sleep disturbances affect a variety of physiological systems, including the immune, endocrine, and circulatory systems. A negative impact on these systems increases the risk of various illnesses, such as diabetes, high blood pressure, cancer, and infections. Accordingly, Yun and Jo [2] argued that insomnia should be considered a systemic disease, and they described in detail the complex, dynamic, and global biological nature of the sleep process.

Sleep plays an important role in adults but is also important for child development. Sleep is a necessary physiological process for typical synaptic development and brain maturation, and children with sleep disturbances are vulnerable to emotional and cognitive developmental problems [5]. Seo [3] reviewed in detail the sleep disturbances seen in ASD among the neurodevelopmental disorders that mainly occur in children. The developmental and behavioral effects of sleep disturbances in ASD were examined in detail, and the underlying mechanisms were investigated. In addition, he looked at the therapeutic approaches to sleep disturbances in children and adolescents with ASD, including pharmacological and non-pharmacological treatments.

Nevertheless, new attempts are being made to treat sleep disorders. Sleep medications commonly used for sleep disorders have many side effects and therapeutic limitations. Kim [4] reviewed the effects of mindfulness meditation, which has recently been in the spotlight, on insomnia. Patients with chronic insomnia describe their condition as a "vicious cycle." In other words, insomnia worsens as the effort and desire to sleep increases. These cognitive distortions and excessive sleep obsessions cannot be resolved with medication alone. The concept and therapeutic mechanism of mindfulness meditation that is helpful in patients

Copyright (C) 2021 Yeungnam University College of Medicine

This is an Open Access article distributed under the terms of the Creative Commons Attribution Non-Commercial License (http://creativecommons.org/licenses/by-nc/4.0/) which permits unrestricted non-commercial use, distribution, and reproduction in any medium, provided the original work is properly cited. 
with chronic insomnia will be examined in detail.

I hope these articles will help clinicians and scientists better understand sleep and insomnia. These articles are expected to be helpful for a comprehensive understanding of sleep. The systemic characteristics of sleep were examined through physiological mechanisms, and the sleep patterns of children with ASD, which have been addressed little in the past, were examined in depth. A mindfulness-based insomnia treatment, a non-pharmacological treatment that has recently been in the spotlight, was also described in detail.

Finally, I would also like to thank the Editorial Board of Yeungnam University Journal of Medicine for giving us the opportunity to conduct a multifaceted review of sleep. I appreciate the hard work of the authors of these articles.

\section{Notes}

\section{Conflicts of interest}

No potential conflict of interest relevant to this article was reported.

\section{ORCID}

Hye-Geum Kim, https://orcid.org/0000-0002-9677-7011

\section{References}

1. Singareddy R, Vgontzas AN, Fernandez-Mendoza J, Liao D, Calhoun S, Shaffer ML, et al. Risk factors for incident chronic insomnia: a general population prospective study. Sleep Med 2012;13:346-53.

2. Yun S, Jo S. Understanding insomnia as systemic disease. Yeungnam Univ J Med 2021;38:267-74.

3. Seo WS. An update on the cause and treatment of sleep disturbance in children and adolescents with autism spectrum disorder. Yeungnam Univ J Med 2021;38:275-81.

4. Kim HG. Effects and mechanisms of a mindfulness-based intervention on insomnia. Yeungnam Univ J Med 2021;38: 282-8.

5. Hirata I, Mohri I, Kato-Nishimura K, Tachibana M, Kuwada A, Kagitani-Shimono K, et al. Sleep problems are more frequent and associated with problematic behaviors in preschoolers with autism spectrum disorder. Res Dev Disabil 2016;49-50:86-99. 